\title{
Quaderni
}

QUADERN I Communication, technologies, pouvoir

67 | Automne 2008

Jeu vidéo et discours

\section{Du rôle de la masculinité militarisée dans la médiation ludique sur support numérique}

The role of militarized masculinity in playful mediation in digital entertainment

\section{Sébastien Genvo}

\section{(2) OpenEdition}

\section{Journals}

Édition électronique

URL : http://journals.openedition.org/quaderni/282

DOI : 10.4000/quaderni.282

ISSN : 2105-2956

\section{Éditeur}

Les éditions de la Maison des sciences de l'Homme

\section{Édition imprimée}

Date de publication : 5 octobre 2008

Pagination : 43-52

\section{Référence électronique}

Sébastien Genvo, «Du rôle de la masculinité militarisée dans la médiation ludique sur support numérique », Quaderni [En ligne], 67 | Automne 2008, mis en ligne le 05 janvier 2012, consulté le 01 mai 2019. URL : http://journals.openedition.org/quaderni/282 ; DOI : 10.4000/quaderni.282 


\section{$D$ ossier}

\section{du rôle de la} masculinité militarisée dans la médiation ludique sur

\section{support numérique}

\section{Sébastien \\ Genvo}

Maître de conférences en sciences de l'information et de la communication

Université de Limoges Centre de recherches sémiotiques
Identifié par le chercheur Stephen Kline dans l'ouvrage Digital Play sous le terme de « masculinité militarisée », un ensemble de représentations dominantes traversent les productions vidéoludiques depuis la naissance de cette industrie. Cette notion renvoie notamment à « un réseau sémiotique partagé tournant autour de sujets de guerre, de conquête et combat $»^{1}$ (Kline, Dyer-Witheford, De Peuter, 2003 : 255). Dès lors, il nous semble qu'il y a ici une nécessité de comprendre les raisons de la présence de ces représentations dans les processus de « médiation ludique » instaurée par l'industrie du jeu vidéo, le concept de médiation ludique étant ici à comprendre comme un phénomène de diffusion de formes symboliques destinées à produire une signification partagée de jeu au sein d'une communauté (voir Genvo, 2008). Afin de répondre à ce questionnement, nous proposons dans un premier temps de revenir sur les travaux de Stephen Kline afin de formuler par la suite un modèle d'analyse permettant de décrire les différents niveaux d'expression de ces logiques au sein d'un jeu vidéo. Comme nous le verrons, pour répondre à notre interrogation initiale, il sera nécessaire de faire la distinction entre les modalités d'interaction ludique - qui entretiennent un rapport naturel à l'action et à la compétition dès lors que l'individu poursuit un objectif dans un cadre ludique - et les mises en scène de ces interactions, qui révèlent un certain rapport de réflexivité vis-à-vis de l'industrie globalisée qui les produit.

Une représentation culturelle dominante dans l'histoire vidéoludique

Selon nous, pour comprendre le rôle de la masculinité militarisée dans la médiation ludique 
sur support numérique, il est tout d'abord nécessaire de considérer ce « réseau sémiotique partagé » en tant que représentation culturelle du jeu, bien que ce terme ne soit pas employé par Stephen Kline. Pour justifier ce choix, il nous faut développer plus en avant la définition de la notion de représentation culturelle, en nous fondant sur la réflexion que propose Dan Sperber (1999 : 133-134) : «Toute représentation met en jeu une relation entre au moins trois termes: la représentation elle-même, son contenu, et un utilisateur, trois termes auxquels peut s'ajouter un quatrième : le producteur de la représentation lorsque celui-ci est distinct de l'utilisateur ; il $s$ 'agit alors d'une représentation mentale. Un souvenir, une hypothèse, une intention sont des exemples de représentations mentales. L'utilisateur et le producteur d'une représentation mentale ne font qu'un. Une représentation peut aussi exister dans l'environnement de l'utilisateur comme par exemple le texte qui est sous vos yeux ; il s'agit alors d'une représentation publique. Une représentation publique est généralement un moyen de communication entre un producteur et un utilisateur distincts de l'autre. [...] Parmi les représentations communiquées, certaines - une très petite portion - sont communiquées de façon répétée et peuvent même finir par être distribuées dans le groupe entier, c'est-à-dire faire l'objet d'une version mentale dans chacun de ses membres. Les représentations qui sont ainsi largement distribuées dans un groupe social et l'habitent de façon durable sont des représentations culturelles. Les représentations culturelles ainsi conçues sont un sous-ensemble aux contours flous de l'ensemble des représentations mentales et publiques qui habitent un groupe social ». À la suite de cette définition, il est possible d'avancer, selon le postulat de l'auteur de Digital Play, que les jeux vidéo véhiculeraient majoritairement et de façon durable des représentations publiques du jeu dont le contenu a trait à des sujets de guerre et de conquête, ces représentations étant alors diffusées largement, à l'échelle mondiale dans le cas de l'industrie vidéoludique.

Dès lors, il est nécessaire d'expliquer les causes de ce type de représentations afin de comprendre quelles seraient les raisons de leur pérennité : " expliquer le caractère culturel de certaines représentations, c'est répondre à la question suivante: pourquoi ces représentations sontelles plus contagieuses et réussissent-elles mieux que d'autres dans une population humaine donnée? (Sperber, 1999 : 145). Un des facteurs à prendre en considération pour comprendre ce qui a déterminé la formation de cette représentation est tout d'abord d'ordre technologique (au sens d'appropriation d'une technique). Les jeux vidéo ont été initialement développés sur des machines conçues pour faciliter la modélisation de situations de guerre. Spacewar, développé en 1962 et souvent considéré comme le premier jeu vidéo de l'histoire, est par exemple issu des expérimentations d'un étudiant du MIT (qui faisait partie du mouvement hacker), dans un contexte de guerre froide où les instituts de recherche technologique étaient largement subventionnés par des fonds de soutien en provenance d'organismes comme l'agence des projets de recherche avancée ${ }^{2} \mathrm{du}$ Pentagon. Ce contexte de naissance a joué un rôle important par la suite dans le développement de l'industrie, l'armée ayant toujours supporté le secteur pour la conception de certaines de ses technologies. Déjà en 1980, le jeu Battlezone (Atari), un simulateur de tank, avait été racheté 
par l'armée américaine dans une version modifiée, ceci dans le but d'entraîner ses soldats. La liste des logiciels ayant fait l'objet de ce genre de rachat ne cesse alors d'augmenter. En 2002, l'armée américaine développe son propre jeu, America's Army, disponible gratuitement par téléchargement sur un site internet dédié3 ${ }^{3}$.

Mais, il est également possible d'avancer que la prégnance de « modèles » de jeu empreints de masculinité militarisée a une cause marketing. Cet aspect a réellement commencé à se développer à grande échelle avec la montée en puissance de Nintendo au milieu des années 80 , qui voulait répondre aux attentes de son public cible majoritaire, des garçons préadolescents. À ce titre, l'offensive de Sega au début des années 90 , voulant toucher un public plus «mature», s'est traduite par une intensification de ce type de représentations au sein des jeux et dans les campagnes promotionnelles (voir Bruno, 1993). L'augmentation des coûts de développement, des campagnes de marketing et l'ascendance des constructeurs de console sur la chaîne de production (qui imposaient aux développeurs une certaine politique éditoriale par la validation des contenus) n'incitèrent pas les éditeurs de jeux à sortir des sentiers battus qui avaient permis la croissance de cette industrie globalisée.

Mais trouver les causes de la présence de ce type de représentations culturelles ne permet pas d'expliquer en quoi celles-ci réussissent mieux que d'autres dans une population humaine donnée, pour reprendre les termes de Dan Sperber. Sur ce point Stephen Kline ébauche le début d'une piste à suivre : « la violence est un idiome culturel qui ne requiert pas de traduction à l'intérieur des marchés transnationaux du divertissement sans cesse croissants : les jeux d'arts martiaux, par exemple, peuvent traverser le pacifique du Japon jusqu'aux États-Unis et revenir à nouveau très facilement $"{ }^{4}$ (Kline, Dyer-Witheford, De Peuter, 2003 : 251). Selon nous, ce dernier aspect interroge une acception particulière du terme de représentation au sein de la culture vidéoludique. Il s'agit d'une forme de savoir pratique qui institue auprès du joueur un capital culturel ludique spécifique, qui sert essentiellement «à l'ajustement pratique $d u$ sujet à son environnement $»^{5}$ et facilite la reproduction de schèmes préconstruits et partagés. À travers des thématiques de conquêtes, de conflits, l'industrie vidéoludique diffuserait donc des représentations personnelles et sociales qui concourraient à un rôle de boussole au sein de l'environnement ludique, les jeux sur support numérique étant susceptibles de désorienter l'utilisateur du fait de la variation perpétuelle des modalités d'interactions depuis la naissance du médium (ceci se traduit notamment par une multiplication et une hybridation récurrente des genres de jeux vidéo, voir à ce sujet l'article de Matthieu Letourneux, 2005).

Toutefois, si cette représentation culturelle peut être décelée dans un très grand nombre de productions vidéoludiques, il nous semble nécessaire de revenir sur les façons dont elle prend effectivement forme au sein du médium. Stephen Kline présente à juste titre Super Mario Bros. comme l'un des jeux phares ayant permis à l'industrie de concrétiser son expansion - au début des années 80 Nintendo a notamment réussi à reprendre le flambeau d'Atari sur le plan économique après une importante crise ayant touché le secteur des consoles de «salon ». Doit-on néanmoins 
qualifier de la même sorte ce jeu, qui repose certes sur une activité de conquête (voire de « colonisation » d'un territoire, pour reprendre le terme employé par l'auteur de Digital Play), et des jeux de tir en vue subjective comme Doom ou Quake, autres productions emblématiques de la masculinité militarisée pour Kline ? Le médium vidéoludique, du fait de son interactivité, ne procède-t-il pas intrinsèquement d'une dynamique d'incitation à l'action ? À quel moment entre-t-on alors dans un cadre de masculinité militarisée, induisant des modalités d'interaction ludique reposant sur la violence?

\section{Différents niveaux de mise en scène du conflit ludique}

Dans leur ouvrage L'univers des jeux vidéo (1998), Alain et Frédéric Le Diberder minimisent la part de jeux pouvant finalement être considérés comme « violents » dès lors que l'on procéderait à une distinction entre le contexte narratif ou symbolique du jeu et les actions demandées au joueur. En répertoriant par exemple les vingt jeux issus des meilleures ventes de 1993, les auteurs précisent pour chacun le genre auquel ils appartiennent et constatent finalement que seulement deux jeux ${ }^{6}$ pourraient être considérés comme violents, qualifiant notamment quatre jeux comme simulateurs militaires ${ }^{7}$ sans les comptabiliser pour autant comme violents. Ceci leur permet de déclarer que «les jeux violents sont une proportion nettement minoritaire et en déclin des jeux, tant offerts qu'utilisés. [...] On ne devrait pas utiliser les mêmes mots pour évoquer la violence d'un jeu selon qu'il invite le joueur à des actions violentes dans un contexte explicitement violent, comme dans un combat de rue, ou dans un contexte non violent, comme dans un jeu de sport. De quelle nature est par exemple la violence d'un wargame? Le joueur y passe son temps à réfléchir. On ne lui demande ni vitesse d'exécution ni adresse, mais simplement d'avoir une bonne stratégie. Le cadre du jeu est cependant celui de centaines, voire de milliers de morts, au service d'un but guerrier. La violence est symbolique, presque jamais représentée, et toujours en respectant les conditions exposées par Computer Gaming World : pas de violence gratuite.» (Le Diberder, 1998 : 187-188).

Ce type de discours sur l'euphémisation de la violence dans les jeux vidéo requière bien sûr une importante réserve, dans la mesure où une violence « symbolique » peut tout à fait conduire à un vécu problématique pour le joueur.

Pour reprendre le cas des «wargames», Michel Nachez et Patrick Schmoll ont étudié les formes et significations de l'incivilité dans les jeux en ligne en se fondant plus particulièrement sur le rôle des « Player Killers $»^{8}$ dans le jeu de stratégie à univers persistant ${ }^{9}$ Mankind. Ils constatent alors que « les formes de la violence évoluent vers davantage de réalisme : un réalisme qui, là non plus, n'est pas un effet des prouesses de l'imagerie infographique, mais de ce flou des frontières entre la fiction et la réalité, l'espace de jeu et celui de la vie quotidienne. La violence n'est plus seulement un spectacle d'animation, elle est vécue, effectivement infligée ou subie. Elle n'est pas physique, bien sûr, dans un monde virtuel. Mais se faire dérober ou détruire des artefacts ou des installations, même virtuels, qu'on a mis des semaines ou des mois à construire provoque des émotions qui sont bien réelles. Contraire- 
ment aux jeux de massacre qui donnent lieu à une sociabilité bon-enfant, l'expérience dans les “mondes persistants" d'agressions vécues comme telles peut susciter, non seulement la colère ou le découragement, mais la haine. Et au noyau de cette haine, trône la figure, ô combien vilipendée, du Player Killer» (Nachez, Schmoll, 2002 : 85).

Ces éléments de réflexion incitent à prendre en compte les différents niveaux d'expression de masculinité militarisée dans la médiation ludique qu'instaure un jeu vidéo. Dans une optique ludologique ${ }^{10}$, Gonzalo Frasca recommande de discerner trois niveaux distincts au sein desquels peut s'exprimer une idéologie (entendue comme système de représentation). Le premier est celui de l'histoire, des événements mis en jeu : « ceci inclut les caractéristiques des objets et personnages, le contexte, les arrangements, et les scènes cinématiques. Par exemple, un simple changement d'apparence des personnages peut transformer Quake en un match à mort entre Israéliens et Palestiniens ${ }^{11}$ (Frasca, 2003 : 232). Nous pouvons compléter cette analyse en avançant que c'est l'ensemble des connaissances déclaratives qui constituent le jeu qui est à prendre en compte à ce niveau. Le second niveau se situe dans la manipulation des règles. En somme, ce que le joueur peut faire à l'intérieur du modèle de jeu. Est-il libre par exemple d'attaquer d'autres participants dans un jeu en ligne sans que cela ne trouve de justification vis-à-vis des objectifs qui lui sont assignés ? Le dernier niveau concerne enfin les objectifs à atteindre, ce que le jeu propose au joueur de faire pour progresser. Doit-il délivrer une princesse (comme dans Super Mario Bros.) ou massacrer les habitants d'un village atteints par une étrange maladie (Resident Evil 4) ? Ces différents niveaux sont bien sûr en interaction, pouvant véhiculer de concert un même message, ou alors faire naître des contradictions.

\section{Un impératif de l'action : une modalité de médiation problématique?}

Comme nous l'avons souligné par ailleurs, il est possible de considérer qu'un conflit apparaît dès lors que l'individu joue à un jeu ${ }^{12}$. En tentant d'accomplir les buts qu'il s'impose, le joueur entrera au minimum en compétition avec le système de règles, qui doit maintenir une certaine incertitude sur le résultat de l'action pour s'inscrire dans le cadre du jeu ${ }^{13}$. Dès lors, puisque le jeu vidéo incite à agir dans un cadre ludique, un rapport naturel à l'action et au conflit s'instaure. Les différents points d'analyse présentés précédemment permettent alors de mieux cerner la façon dont on pourrait qualifier la masculinité militarisée dans ces productions. Même si, pour reprendre les termes de Stephen Kline, Mario peut être considéré comme un colonisateur (le but du jeu est de progresser au sein d'un territoire), le répertoire d'actions mis à disposition du joueur au sein de l'univers ludique (il faut sauter de plateformes en plateformes, éliminer ses opposants par un saut, etc.), et le monde de référence de celui-ci (emprunt de shintoïsme, d'esprits de la nature), diffèrent trop d'un jeu comme Doom (où il faut affronter des démons à coup de fusil à pompe et de tronçonneuse pour sortir d'un complexe militaire) pour qu'il soit envisageable de les affilier de la même façon au « réseau sémiotique partagé » identifié par Kline, ceci même si l'objectif est semblablement le même. Le succès d'un jeu tel que Super Mario Bros., et 
son rôle dans l'expansion de l'industrie, tendrait donc à modérer l'importance que peut revêtir la masculinité militarisée dans les productions vidéoludiques. Il ne faut pas oublier néanmoins qu'il s'agit selon nous d'une représentation culturelle dominante, qui peut certes rencontrer des frictions, des contradictions, voire entraîner des résistances, mais dont l'hégémonie n'a jamais été remise en cause. Alors que Nintendo était en position de quasi monopole dans le marché des consoles au cours des années 80 , la concurrence de Sega - qui souhaitait donner une image infantile, voire immature de son concurrent - s'est traduite par une réaction assez ambiguë du premier. Tout en prônant la défense de valeurs familiales chères à son public initial, de nombreux titres de la nouvelle console de Nintendo, la « Super NES », étaient des adaptations de jeux d'arcade particulièrement violents (le titre le plus représentatif étant le jeu photo-réaliste Mortal Kombat de Acclaim Entertainment).

Est-ce pour autant l'impératif d'action du jeu vidéo qui encourage les éditeurs à conforter cette tendance dans leur stratégie de développement, ou est-ce avant tout le public cible majoritaire de ce type de produit qui a conduit au développement de cette thématique, pour anticiper la demande ? Dans ce dernier cas, les nécessités de diversification des publics entraînées par l'augmentation des coûts de production (avec la saturation du marché des adolescents et jeunes adultes masculins) pourrait laisser envisager une future remise en cause des représentations empreintes de masculinité militarisée dans les productions de l'industrie vidéoludique, à l'image des Sims, succès mondial majoritairement joué par des filles. Toutefois, l'analyse que fait Bernard
Perron sur les émotions dans les jeux vidéo laisse à penser que les raisons sont internes au médium vidéoludique : «Les notions de contrôle (ou la perte de celui-ci) et d'action sont au cour de l'expérience du jeu vidéo. On comprend alors pourquoi la peur demeure l'émotion vidéoludique la plus exploitée. Elle est clairement orientée vers un objet et vers un but. C'est une émotion primaire et prototypique qui a une forte tendance à l'action » (Perron, 2005 : 360).

Est-ce à dire que certaines émotions seraient à exclure du champ de l'expression vidéoludique ? Par exemple, la tristesse ne rendrait-elle pas trop apathique pour conforter le joueur dans une dynamique de l'action? Pour explorer d'autres horizons émotionnels, certains jeux cherchent à complexifier l'impératif d'action. Un jeu comme Ico fonde par exemple ses mécanismes de jeu sur un sentiment d'inquiétude pour autrui (Chauvin, 2002) et non de peur pour soi-même. De même l'étude de Michel Nachez et Patrick Schmoll sur le parcours ludique des « Player Killers » montre la « capacité des acteurs à changer, en l'occurrence à assimiler la dimension de l'autre, impliquant une modification de la logique de leurs interactions au sein du système. Les incivilités seraient ainsi porteuses à la fois de destruction et de sens. Elles ne constitueraient qu'un passage limité par la négativité dans un processus plus général de socialisation » (Nachez, Schmoll, 2002 : 91). Mais les précédentes réflexions révèlent qu'au final un prisme de facteurs, à la fois structurels et contextuels, concourent encore aujourd'hui à conforter à divers degrés la pérennité des représentations empreintes de «masculinité militarisée » dans les modalités de médiation ludique mises en place par les jeux vidéo. 


\section{De la nécessité d'une approche critique}

Si l'on commence l'histoire des jeux vidéo à Spacewar en 1962, il est possible de considérer que ceux-ci ont aujourd'hui plus de 40 ans d'existence. Lorsqu'il s'agit de montrer l'ampleur économique de ce «nouveau » médium, il est assez usuel de le comparer économiquement avec le cinéma : les analystes avancent habituellement que les jeux vidéo génèrent un chiffre d'affaires supérieur à celui du cinéma en salle (cette comparaison étant bien sûr hautement symbolique). Mais si l'on compare le jeu vidéo au cinéma des années 30 en terme de contenus et de thématiques abordés, il est probant que le domaine souffre encore d'une certaine immaturité. Il est bien difficile de trouver des jeux qui proposent au joueur une réflexion critique et originale sur notre monde, tout en répondant à des impératifs de divertissement et de rentabilité économique. En somme, il manque encore au domaine des œuvres critiques de la portée d'un film comme Les Temps modernes. Depuis la naissance de l'industrie vidéoludique, plusieurs jeux en marge des grands circuits de distribution ont néanmoins tenté de proposer une alternative aux logiques dominantes, un des derniers exemples en date étant September $12 t h^{14}$ de Gonzalo Frasca. Mais ces jeux restent encore largement « à la marge », et ne suscitent pas de modèles économiques viables, stables et durables. Comme le relève Stéphane Natkin (2008), les concepteurs de titres indépendants des circuits habituels de distribution sont le plus souvent embauchés par des studios plus importants sans que le jeu qui a permis leur reconnaissance ne fasse pour autant l'objet d'une diffusion ou d'une distribution de plus grande ampleur. Ceci serait pourtant à même de rela- tiviser l'hégémonie d'une certaine masculinité militarisée dans les productions vidéoludiques. La réussite économique sporadique de jeux s'écartant quelque peu de ce type de représentation du monde, tel Ico, laisse toutefois entrevoir la possibilité d'un inversement de tendance. Le développement d'une éducation au multimédia et d'un regard critique chez les utilisateurs et les développeurs (des formations aux métiers du domaine devenant de plus en plus fréquentes) est selon nous une des voies à emprunter pour engager cette maturité du médium. À ce titre, le présent article a souhaité donner quelques points de repères pour entreprendre cette vaste exploration. 
$R \cdot \dot{E} \cdot F \cdot \dot{E} \cdot R \cdot E \cdot N \cdot C \cdot E \cdot S$

BRUNO Pierre, Les jeux vidéo, Paris, Syros, 1993.

CAILLOIS Roger, 1967, Les jeux et les hommes, Paris, Gallimard, 1958.

CHAUVIN Jean-Baptiste, « Ico. Solitudes », Cahiers du cinéma, hors-série, septembre, 2002.

FRASCA Gonzalo, « Simulation versus narrative », pp. 221-235, in : Wolf Mark.J.P., Perron Bernard, dirs., The video game theory reader, New York, Routledge, 2003.

GENVO Sébastien, Le game design de jeux vidéo : approche communicationnelle et interculturelle, Thèse en sciences de l'information et de la communication, Université Paul Verlaine - Metz, disponible en ligne : http://www.ludologique. com/publis/these.html, 2006

GENVO Sébastien, « Réflexions ludologiques » in : Genvo Sébastien, Coord., Les jeux vidéo, un «bien » culturel ?, Médiamorphoses, 22, INA, 2008.

KLINE Stephen, DYER-WITHEFORD Nick, DE PEUTER Greig, Digital Play, Montreal \& Kingston, McGill-Queen's University Press, 2003.

LE DIBERDER Alain, LE DIBERDER Frédéric, L'univers des jeux vidéo, Paris, La découverte, 1998.

LETOURNEUX Matthieu, «La question du genre dans les jeux vidéo », in : Genvo Sébastien, dir., Le game design de jeux vidéo. Approches de l'expression vidéoludique, Paris, L'Harmattan, 2006.

MUCCHIELLI Alex, Les méthodes qualitatives, Paris, Presses universitaires de France, 1994.
NATKIN Stéphane, «Une école pour les jeux vidéo » in : Genvo Sébastien, Coord., Les jeux vidéo, un «bien » culturel?, Médiamorphoses, 22, INA, 2008.

NACHEZ Michel, SCHMOLL Patrick, « Les Player Killers. Formes et significations de l'incivilité dans les jeux vidéo en ligne », Revue des sciences sociales, 29, 2002.

PERRON Bernard, " Jeux vidéo et émotions », in : Genvo Sébastien, dir., Le game design de jeux vidéo. Approches de l'expression vidéoludique, Paris, L'Harmattan, 2006.

SPERBER Dan, «L'étude anthropologique des représentations : problèmes et perspectives », in Jodelet Denise, Dir., Les représentations sociales, Presses universitaires de France, 1999. 
$\mathrm{N} \cdot \mathrm{O} \cdot \mathrm{T} \cdot \mathrm{E} \cdot \mathrm{S}$

1. Notre traduction de l'anglais.

2. Usuellement dénommée ARPA soit Advanced Research Projects Agency.

3. <http://www.americasarmy.com/>, consulté le 06/12/05.

4. Notre traduction de l'anglais.

5. « $\grave{A}$ un autre niveau la représentation est une "forme de savoir pratique", une sorte de modélisation et d'intégration personnelle ou sociale des informations possédées sur un sujet. Ce savoir sert essentiellement "à l'ajustement pratique du sujet à son environnement" " (Mucchielli, 1994 : 56).

6. Il s'agit de Comanche (Nova Logic), une simulation d'hélicoptère de combat (étrangement qualifié comme jeu d' « arcade » par Alain et Frédéric Le Diberder) et Wolfenstein 3D (Apogée), l'ancêtre de tous les jeux de tir en vue subjective actuels.

7. Il s'agit en fait de trois simulateurs de vol de combat et d'un simulateur de sous-marin se déroulant dans un contexte d'opération de guerre.

8. Un player killer est un joueur qui se fixe comme objectif d'exterminer les autres joueurs sans que ses actes ne trouvent apparemment de justification au sein des règles du jeu.

9. Les jeux en ligne à univers persistant réunissent dans un même monde de jeu des milliers de joueurs, où une partie sans fin se déroule de façon continuelle, que le joueur soit connecté ou non au monde virtuel, qui est en constante évolution. Bien que les règles et mécanismes ludiques puissent différer selon les logiciels en fonction du game design de l'œuvre - du travail mené sur sa structure (règles, buts, etc.) - ces jeux proposent généralement aux utilisateurs de résoudre des quêtes, de se réunir en guilde, de mener des batailles entre diverses factions, etc.
Pour entrer dans ces univers, le joueur devra alors créer un ou plusieurs avatars qui le représenteront à chaque fois qu'il se connectera. Il pourra de même avoir des échanges avec les autres utilisateurs par l'intermédiaire des multiples avatars.

10. Selon Gonzalo Frasca (2003), la ludologie a notamment pour vocation d'analyser dans une optique systémique la structure, les éléments d'un jeu - particulièrement ses règles - et de fonder des typologies et modèles pour expliquer les mécanismes de jeux.

11. Notre traduction.

12. Pour de plus amples détails voir Genvo, 2006, pp. 191-195.

13. Des auteurs tels que Jacques Henriot ou Roger Caillois l'ont relevé, un «déroulement connu d'avance, sans possibilité d'erreur ou de surprise, conduisant clairement à un résultat inéluctable, est incompatible avec la nature du jeu» (Caillois, $1958: 39$ ).

14. Il s'agit d'un jeu développé en flash qui propose une réflexion sur la politique interventionniste des États-Unis. Une version jouable est disponible à ce lien : http://www.newsgaming.com/games/index12. htm 
$R \cdot E ́ \cdot S \cdot U \cdot M \cdot E ́$

Depuis la naissance des jeux vidéo, un ensemble de représentations empreintes de «masculinité militarisée » traversent les productions vidéoludiques. Cette notion renvoie à un réseau sémiotique partagé tournant autour de sujets de guerre, de conquête et de combat. Le présent article propose de comprendre les raisons de la présence de ces représentations dans les processus de « médiation ludique » instaurés par cette industrie. Il s'agit notamment de décrire les différents niveaux d'expression de ces logiques au sein du domaine. Il est proposé de faire la distinction entre les modalités d'interaction ludique - qui entretiennent un rapport naturel à l'action et à la compétition dès lors que l'individu poursuit un objectif dans un cadre ludique - et les mises en scène de ces interactions, qui révèlent un certain rapport de réflexivité vis-à-vis de l'industrie globalisée qui les produit.

The role of militarized masculinity in playful mediation in digital entertainment

Since videogames birth, most of productions are conveying specific representations called « militarized masculinity ». This refers to a shared semiotics network which subject is war, conquest and battle. This article proposes to understand the reasons of these representations presence in the process of « playful mediation » established by the industry. These include in-field description of these logics' different levels of expression. Then distinction between playful interaction modalities - which have a natural action and competition if the individual pursues a goal in a playful frame - and the staging of these interactions would reveal a certain reflectivity vis-à-vis the global industry that produces them. 